\title{
Upper Gastrointestinal Surgery: Robotic Surgery versus Laparoscopic Procedures for Esophageal Malignancy
}

\author{
Matthias Biebl Andreas Andreou Sascha Chopra Christian Denecke Johann Pratschke \\ Department of Surgery, Campus Klinikum Virchow and Campus Charité Mitte, Charité - University Medicine Berlin, Berlin, Germany
}

Keywords

Esophageal cancer - Minimally invasive esophagectomy . Robotic-assisted minimally invasive esophagectomy . RAMIE $\cdot$ Hybrid resection

\section{Summary}

Background: The evolution of minimally invasive surgery (MIS) also extends to the field of esophageal surgery and has brought forth the development of several approaches of minimally invasive esophagectomy (MIE). Hybrid and total minimally invasive operative techniques have proven beneficial compared to open surgery and are currently evaluated against robotic-assisted minimally invasive esophagectomy (RAMIE). We aim to review the current literature regarding the position of MIE versus RAMIE. Methods: A systematic review of the relevant literature on minimally invasive esophageal surgery for cancer is presented. A PubMed search was carried out for the period of 19922018 with the following search terms: 'esophageal cancer', 'minimally invasive surgery', 'resection', 'transhiatal', 'transthoracic', 'MIE', 'hybrid', 'robotic resection', 'RAMIE', 'RATE'. Results: Hybrid and total minimally invasive operative techniques have proven beneficial, especially with regard to pulmonary complications, compared to open surgery. Oncologic outcomes appear equivalent between open and minimally invasive techniques. Currently, the position of RAMIE is being evaluated against other minimally invasive techniques. Conclusion: All minimally invasive techniques confer the expected reduction in perioperative morbidity compared to open surgery. However, MIS is still evolving with regard to specific technical challenges, especially anastomotic techniques.

(C) 2018 S. Karger GmbH, Freiburg

\section{Introduction}

Esophageal cancer is a devastating disease with a mortality rate approaching tumor incidence. In 2012, an estimated 455,800 esophageal cancer cases and 400,200 cancer-related deaths were reported, accounting for the 6th highest cancer mortality worldwide $[1,2]$. Two predominant types of cancer occur: squamous cell cancer (SSC) and adenocarcinoma (AC). Both types differ with regard to pathogenesis, epidemiology, tumor biology, and treatment regimens, with SSC being the more common histology [1]. SSCs are often located higher up in the esophagus (mainly mid-esophagus), while AC are more commonly found close to the esophagogastric junction. Due to the often late diagnosis, radical treatment is undertaken in only 20 $30 \%$, with long-term survival after curative-intended therapy being approximately $25 \%[3,4]$.

Given this dismal situation, the standard treatment with curative intent for advanced tumors ( $\mathrm{cT} 3$ and/or $\mathrm{cN}+$ ) of the esophagus or esophagogastric junction includes a multimodal approach. For SSC, preoperative radiochemotherapy is followed by radical tumor resection [5-7]. For AC, preoperative radiochemotherapy may - besides perioperative (pre- and postoperative) chemotherapy - also be a standard treatment prior to surgical resection [8-11].

Curative surgery includes radical resection of the tumor-bearing segment of the esophagus and the proximal stomach together with radical lymphadendectomy in the abdomen around the celiac trunk and the lower and mid-mediastinum (2-field lymphadenectomy). While tumors of the esophagogastric junction are resected via either a transhiatal or a transthoracic approach $[12,13]$, for higher-up tumors, transthoracic resection with either an intrathoracic (IvorLewis) or a cervical anastomosis (McKeown) $[14,15]$ is performed. While in early experiences, an en-principe cervical anastomosis was often accepted due to better conservative management options in the case of anastomotic leakage (reported in 0-35\% after resection) [16], a higher anastomosis results in higher leakage rates, more anastomotic strictures, more dysphagia, and a decreased quality of life compared to an intrathoracic anastomosis $[15,17]$. With modern interventional endoscopic treatment options [18], intrathoracic anastomosis is becoming more attractive and many centers have adopted

\section{KARGER}

() 2018 S. Karger GmbH, Freiburg

Fax +497614520714
Prof. Dr. med. univ. Matthias Biebl Department of Surgery

Charité - University Medicine Berlin, Campus Klinikum Virchow

Augustenburger Platz 1, 13353 Berlin, Germany matthias.biebl@ charite.de 


\section{Degree of invasiveness during esophageal resection}

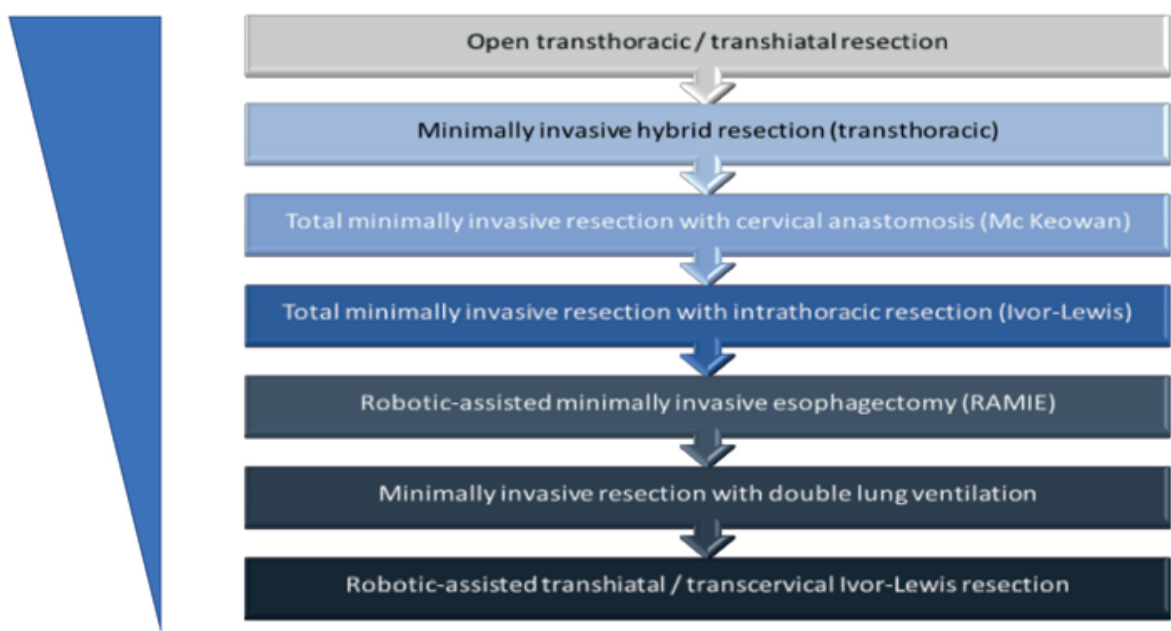

transthoracic resection and re-anastomosis as their standard approach, especially in Western countries where more distal tumors are treated.

Mortality rates of esophageal resection are expected to be below $5 \%$; however, postoperative morbidity may be as high as 50\%, mainly due to pulmonary complications [12]. Consequently, only about 50$60 \%$ of patients undergoing surgery after preoperative treatment also receive planned postoperative chemotherapy [19]. Minimally invasive surgery (MIS) could therefore not only reduce the morbidity of the surgical intervention itself, but also may enhance the rate of adherence to planned oncologic treatment following esophageal resection.

\section{Methods}

Recommendations within this review are derived from data from the current literature. A PubMed search was carried out between 1992 and 2018 with the following search terms: 'esophageal cancer', 'minimally invasive surgery', 'resection', 'transhiatal', 'transthoracic', 'MIE', 'hybrid', 'robotic resection', 'RAMIE', 'RATE',

\section{Types of Minimally Invasive Esophageal Resection}

Due to the different approaches to morbidity reduction in esophageal resection, a variety of procedures have been coined 'minimally invasive esophagectomy' (MIE): Most authors relate to a transthoracic resection; however, no semantic differentiation is being made as to whether the anastomosis is performed at the cervical or the intrathoracic level. If both the abdominal and the thoracic part are performed in a minimally invasive fashion, the procedure is named 'total' minimally invasive esophageal resection. Here, the abdominal part is quite uniformly performed laparoscopically with usually 4-6 trocars positioned in a 'smiley' formation at the umbilical level. For the thoracic part, a broad variety of techniques has been described. While some authors prefer a left-sided thoracotomy position with the patient tilted toward the left side (lateral position), a technique with the patient in prone position is also widely used [20]. While the prone position takes advantage of gravity to displace the lung from the dorsal thoracic structures and the esophagus, the lateral position allows for an approach similar to open resection and does not require repositioning of the patient in the case a conversion to open surgery is required during the transthoracic resection phase. Most centers use single-lung ventilation with active $\mathrm{CO}_{2}$ insufflation into the right hemithorax during the thoracic phase; however, the prone position in some patients also allows for double-lung ventilation during the thoracic phase [21]. Respiratory management deserves recognition when discussing MIE, as one of the leading arguments for the introduction of a minimally invasive approach is the reduction of pulmonary complications. Wolfer et al. [22] reported that a capnothorax with a pressure of $8-10 \mathrm{~mm} \mathrm{Hg}$ and an insufflation rate of 2-3 1/min did not significantly influence the respiratory and cardiovascular system. However, acute lung injury occurs in 25$30 \%$ of patients after transthoracic esophagectomy [23], and singlelung ventilation has been implicated in its pathogenesis [24]. Further, the pathophysiologic relevance of capnothorax was also investigated by Mao et al. [25] who found relevant impairment of the coagulatory system in patients after capnothorax. Also, experimental work by Shen et al. [26] demonstrated that changes in pulmonary management with low-volume insufflation significantly reduced postoperative pulmonary complications in patients undergoing esophagectomy with cervical anastomosis. Robotic-assisted minimally invasive esophagectomy (RAMIE) is technically equivalent to total MIE and mainly performed in the setting of single-lung ventilation; however, the steady image and the exact movement of the instruments may further reduce the physiologic alterations during the operation. Figure 1 gives an overview of the available minimally invasive techniques.

\section{Comparison of Minimally Invasive Esophagectomy and Open Resection}

The first description of a transthoracic minimally invasive approach to esophageal resection was reported by Cuschieri et al. [27] in 1992 and via the transhiatal route by Depaula et al. [28] in 1995. Soon after, Cuschieri [29] reported on 26 procedures, 20 performed in lateral and 6 in prone position, and concluded that the prone posi- 
tion might be more advantageous due to reduced pulmonary complications. It then took several years before the new technique was adopted on a larger scale; however, after the year 2000, the number of papers demonstrating technical feasibility rose steeply [17], and several larger series with experiences exceeding 100 patients were published [30, 31]. In 2012, Luketich et al. [17] reported on their large single-center experience with over 1,000 patients undergoing MIE. In their series, the authors reported an excellent 30-day mortality of $1.7 \%$ with an anastomotic leak rate requiring reoperation of $5 \%$, a conversion rate to open resection of $5 \%$, and a median number of 21 lymph nodes retrieved. In their series, the authors performed the first 481 operations using an en-principe cervical anastomosis (McKeown), and then switched to a minimally invasive Ivor-Lewis approach with intrathoracic anastomosis for the second group of patients $(\mathrm{n}=530)$. In the Ivor-Lewis group, the authors found a $0.9 \%$ 30 -day mortality rate (compared to $2.5 \%$ in the McKeown group; $\mathrm{p}=$ 0.083 ) as well as a reduced rate of recurrent laryngeal nerve palsies, less acute respiratory distress syndrome (ARDS), and less congestive heart failure [17]. The principal relevance of avoiding cervical anastomosis whenever possible is also corroborated by a large nationwide registry study of esophageal resection from the USA, demonstrating a significantly higher in-hospital mortality and overall morbidity rate for total esophagectomy compared to partial esophagectomy [32].

Also, the authors reported a postoperative survival comparable to other published data for open resection [17]. In 2015, Luketich et al. [33] published a prospective phase II multicenter trial of 17 US centers including 104 patients. In their study, the authors reported an $8.6 \%$ anastomotic leak rate, an ARDS rate of $5.7 \%$, and a pneumonitis rate of $3.8 \%$, with an estimated 3-year survival rate of $58.4 \%$. The authors concluded that MIE can be adopted and safely performed by other centers proficient in esophageal surgery with appropriate minimally invasive skills. The first prospective randomized trial comparing total MIE to open surgery was published in 2012 (TIME trial) [34]. In the final per-protocol analysis, 50 patients with open resection were compared to 53 patients undergoing minimally invasive resection. Interestingly, in both groups, two-thirds of patients received a cervical anastomosis. The main outcome was a reduction in pulmonary complications at 2 weeks from $29 \%$ (open) to $9 \%$ (MIS group) $(\mathrm{p}=0.005)$ and reduced in-hospital pulmonary infection rates from $34 \%$ (open group) to $12 \%$ (MIS group) $(p=0.005$ ). R0 rate, number of lymph nodes retrieved, and in-hospital and 30-day mortality rate were not significantly different between groups. The conversion rate in the MIS group was $14 \%$, and the reoperation rate was not significantly different but quite high in both groups $(11 \%$ open vs. $14 \%$ MIS). Despite limited patient numbers, this study provided evidence that patients derive relevant benefit from MIS. Several retrospective data have since confirmed these findings [35], including a subgroup analysis which confirmed the significant reduction in pulmonary complications also in elderly patients [36]. The recently published 3-year follow-up of the TIME trial confirmed the appropriateness of a MIS approach compared to open surgery with a $50.5 \%$ 3-year survival in the MIS group (40.4\% in the open cohort, $\mathrm{p}$ $=0.207$ ) [37].

Parallel to a total minimally invasive approach, the hybrid IvorLewis approach with open intrathoracic resection and reconstruction has also become popular. In 2015, Messager et al. [38] reported a large French nationwide retrospective analysis of 663 patients undergoing hybrid MIE compared to 2,346 patients with open resection between 2010 and 2012. Primary outcome was the 30-day postoperative mortality rate, and patients undergoing hybrid resection experienced significantly less mortality both at 30 and at 90 days (3.3 vs. $5.7 \%(\mathrm{p}=0.005)$ and 6.9 vs. $10 \%(\mathrm{p}=0.016)$, respectively). By multivariate analysis, age $>60$ years, preoperative malnutrition, and cardiovascular morbidity were predictive of higher mortality, whereas a hybrid approach was associated with a decrease in mortality $(\mathrm{p}=0.041)$. While this analysis related to a retrospective registry study, Mariette et al. [39] recently reported the 3-year results of a multicenter randomized phase III trial (MIRO trial) comparing hybrid versus open esophagectomy. In 104 open versus 103 hybrid patients, major postoperative morbidity was 64.4 versus $35.9 \%$ (p $<0.001)$, pulmonary morbidity 30.1 versus $17.7 \%(\mathrm{p}=0.037)$, and overall survival at 3 years 54.8 versus $67.0 \%(\mathrm{p}=0.054)$, confirming a substantial survival benefit of hybrid MIE compared to open resection.

Two nationwide registry studies from Japan [40] and the USA [41] corroborated the results in large patient cohorts. The US study conducted propensity score matching (562 MIE patients vs. 562 open patients) and found comparable lymph node numbers ( $\mathrm{p}=$ $0.057), \mathrm{R} 0$ resection rates $(\mathrm{p}=0.323)$, and 30 - and 90 -day mortality ( $\mathrm{p}=0.995$ and $\mathrm{p}=0.848$, respectively) between both groups [41]. The Japanese study retrospectively compared 3,515 MIE patients (total and hybrid MIE combined) with 3,515 matched open resections. The authors found less respiratory complications, blood loss, and surgical site infections in the MIE group, but longer operation times and a higher reoperation rate at 30 days ( 7 vs. $5.3 \%$; $p=0.004$ ) in the MIE group. Operative mortality $(\mathrm{p}=0.414)$ and cancer-specific survival were comparable in both groups [40].

\section{Comparison between Different Minimally Invasive Resection Techniques}

Both a hybrid approach and total minimally invasive resection have proven beneficial in terms of early postoperative parameters compared to open resection $[34,35,39-41]$, and both procedures displayed comparable oncologic results with regard to surrogate parameters (R0 resection rate/lymph nodes retrieved) $[34,39]$ as well as cancer-specific [40] and overall survival [34, 39-41]. With regard to postoperative survival, the MIRO trial [39] was the only prospective randomized study demonstrating a survival benefit at 3 years $(\mathrm{p}=$ 0.05 ) compared to open resection. None of the comparisons of total MIE versus open resection or combined MIE versus open resection confirmed a survival benefit for the MIS technique. Not surprisingly, only scarce data comparing hybrid versus total MIE resections are available as i) differences between different minimally invasive techniques are always less apparent than those seen in comparisons to open resections, and ii) each center has developed and pursued their own minimally invasive approach found to be most applicable. A major argument against pursuing thoracoscopic resection when performing a thoracoscopic anastomosis are technical problems related to the construction of the anastomosis. Further, any center predominantly performing total MIE would still encounter a number of pa- 
tients in whom, for various reasons, a hybrid procedure was performed, implementing a strong bias on a direct comparison. Looking at anastomotic complications in total MIE, prospective data as well as large multicenter data indicate a leakage rate of $9-15 \%[33,34$, 42]. In hybrid resection, the anastomotic leakage rate may be lower $[43,44]$; however, any comparison between studies reporting different technical approaches is difficult due to differences in definitions and in the reporting of postoperative anastomotic complications. 2 studies relate to a direct comparison of a total minimally invasive technique and a hybrid approach: Palazzo et al. [45] reported a series of 172 patients undergoing esophageal resection for cancer. $104 \mathrm{pa}-$ tients underwent total MIE (92.3\% with cervical anastomosis), and 68 patients not suitable for such an approach underwent open surgery or a hybrid procedure (combined group). In these strongly selected groups, minor $(\mathrm{p}=0.05)$ and major $(\mathrm{p}<0.01)$ complications as well as the pneumonia rate $(\mathrm{p}=0.01)$ were significantly higher, and the postoperative estimated survival was lower $(\mathrm{p}<0.01)$ in the combined group of open and hybrid resections as compared to the MIE cohort. Bonavina et al. [46] published a series of 80 total MIE versus 80 hybrid MIE patients and found no differences in early postoperative complications and mortality.

\section{Robotic-Assisted Minimally Invasive Esophageal Surgery}

After its implementation in other surgical fields, the robot was also evaluated for esophageal surgery. Here, 3-dimensional vision together with the multidimensional articulated movements of the robotic instruments even in narrow spaces [47] seemed attractive to many to overcome the technical difficulties of MIE. As in open surgery, both the transhiatal (robotic-assisted transhiatal esophagectomy (RATE)) (2003) [48] and the transthoracic route (RAMIE) (2004) [49] have been evaluated. Given the results of oncologic esophagectomy, also in robotic surgery, the transthoracic route became the most commonly used approach. Similar to MIE, different variations of RAMIE have also been established, and the principal advantages of the minimally invasive approach compared to open surgery also apply to the robotic-assisted techniques. While with the evolution of robotic techniques fully-robotic approaches emerged, initial series often focused on robotic thoracoscopic dissection with either open or laparoscopic gastrolysis and cervical anastomosis [50]. Ruurda et al. [51] summarized the first 300 published cases in 2015, with no series exceeding 52 cases. The authors concluded RAMIE to have technical feasibility and good short-term oncologic outcomes. However, it was also stated that - unlike MIE or open resection - in RAMIE, the preferred approach to anastomosis was unclear and the reported leak rates varied significantly. Still, the most commonly used anastomotic site was cervical [51]. In a series of 85 patients, Cerfolio et al. [52] performed a laparoscopic gastrolysis, robotic thoracic dissection, and intrathoracic reconstruction with a S/S $30-\mathrm{mm}$ stapled anastomosis and hand-sewn closure of the stapler insertion site, reporting a $7.1 \%$ anastomotic leakage rate and a 3.5 and $10.6 \%$ 30- and 90-day mortality rate, respectively. Abott et al. [53] evaluated a cohort of 134 patients and found the complication rate in patients $>70$ years of age undergoing RAMIE to be comparable to that in younger patients. Also, the robot proved beneficial especially in obese patients, and no differences in operation time, postoperative complications, and length of hospital stay were noted between normal-weight, overweight, and obese patients in a series of 129 patients reported by Salem et al. [54].

Further standardization and the introduction of the latest generation of robotic systems resulted in progression to a fully robotic transthoracic procedure. The most commonly performed anastomotic techniques are hand-sewn at the cervical level [55], and either stapled S/S esophagogastrostomy [52] or a robotic hand-sewn E/E esophagogastrostomy [56].

Early and long-term oncologic outcomes of RAMIE are comparable to other resection techniques $[41,55]$.

Regarding the use of RAMIE, in a US nationwide experience, Weksler and Sullivan [41] reported a National Cancer Data Base (NCDB) analysis of the years 2010-2013. Out of 9,217 esophagectomies, $32.1 \%$ were performed in a minimally invasive fashion. Of these 2,960 resections, 581 (19.6\%) were completed as RAMIE.

\section{Comparison of Robotic and Minimally Invasive Esophagectomy}

While no differences in postoperative mortality and disease-free survival between MIE and RAMIE were noted [41], histopathological data from a study by Park et al. [57] demonstrated that RAMIE allows for a higher number of lymph nodes retrieved compared to MIE. Kim et al. [58] published data on dissection techniques of the upper mediastinum, and Park et al. [59] reported excellent 5-year disease-free (76.2\%) and overall (79.4\%) survival in a cohort of 115 patients undergoing total mediastinal lymphadenectomy. In comparison to the lymph node count reported in large MIE series [17, 31], all RAMIE series applying lymph node dissection of the upper mediastinum [57-59] reported markedly higher numbers of retrieved lymph nodes (median $\mathrm{n}=19-21$ (MIE group) vs. $\mathrm{n}=37-49$ (RAMIE group)).

\section{Special Developments in Robotic-Assisted Esophageal Resection}

In RAMIE, most systems routinely have a real-time perfusion assessment system using diluted indocyanine green, and Hodari et al. [60] reported a low anastomotic leak rate of $6.8 \%$ using this system in a cohort of 54 patients. While RATE itself was not further pursued, interesting developments have been reported using a cervical robotic access for upper mediastinal dissection. Mori et al. [61] performed a preclinical feasibility study using a single-port cervical access for upper mediastinal esophageal dissection. The same group also reported on 22 patients undergoing radical robotic non-thoracic esophagectomy. The authors compared the outcomes with 139 open esophageal resections [62]. Anastomoses in the non-thoracic group were all performed at the cervical level. While procedure time was longer in the non-thoracic group, no statistically significant differences in postoperative morbidity were noted between groups, while the postoperative length of stay was significantly shorter in the minimally invasive group. 


\section{Conclusion}

Comparisons between the different techniques of minimally invasive esophageal resection are difficult due to the variety of techniques and differences in data reporting. Both hybrid and total MIE carry the benefit of reduced morbidity compared to open surgery, with especially pulmonary morbidity being reduced in a minimally invasive setting. Major oncologic outcomes, contraindications to resection, postoperative mortality, and disease-free survival all seem equivalent compared to open standard procedures.

The same applies to robotic-assisted minimally invasive resections. While no direct statistical comparisons are available, the ro- botic approach seems to be more efficient in lymph node retrieval in single-center Asian series compared to large Western experiences with MIE, albeit without clear consequences postoperatively.

The robot offers potential for new dissection routes, minimizing the lung trauma related to the transthoracic approach.

\section{Disclosure Statement}

Matthias Biebl and Johann Pratschke received a research grant from Intuitive Surgery. The other authors report no conflict of interest.

\section{References}

1 Torre LA, Siegel RL, Ward EM, Jemal A: Global cancer incidence and mortality rates and trends - an update. Cancer Epidemiol Biomarkers Prev 2016;25:16-27.

2 Jemal A, Bray F, Center MM, et al: Global cancer statistics. CA Cancer J Clin 2011;61:69-90.

3 MRC Oesophageal Cancer Working Party: Surgical resection with or without preoperative chemotherapy in oesophageal cancer: a randomised controlled trial. Lancet 2002;359:1727-1733.

4 Ychou M, Boige V, Pignon JP, et al: Perioperative chemotherapy compared with surgery alone for resectable gastroesophageal adenocarcinoma: an FNCLCC and FFCD multicenter phase III trial. J Clin Oncol 2011;29:1715-1721.

5 Van Hagen P, Hulshof MCCM, van Lanschot JJB, et al: Preoperative chemoradiotherapy for esophageal or junctional cancer. New Engl J Med 2012;366:2074-2084.

6 Shapiro J, van Lanschot JJB, Hulshof MCCM, et al: Neoadjuvant chemoradiotherapy plus surgery versus surgery alone for oesophageal or junctional cancer (CROSS): long-term results of a randomised controlled trial. Lancet Oncol 2015;16:1090-1098.

7 Ronellenfitsch U, Schwarzbach M, Hofheinz R, et al; GE Adenocarcinoma Meta-Analysis Group: Perioperative chemo(radio)therapy versus primary surgery for resectable adenocarcinoma of the stomach, gastroesophageal junction, and lower esophagus. Cochrane Database Syst Rev 2013;CD008107.

8 Sjoquist KM, Burmeister BH, Smithers BM, et al: Survival after neoadjuvant chemotherapy or chemoradiotherapy for resectable oesophageal carcinoma: an updated meta-analysis. Lancet Oncol 2011;12:681-692.

$\checkmark$ Cunningham D, Allum WH, Stenning SP, et al: Perioperative chemotherapy versus surgery alone for resectable gastroesophageal cancer. N Engl J Med 2006;355: 11-20.

10 Ychou M, Boige V, Pignon JP, et al: Perioperative chemotherapy compared with surgery alone for resectable gastroesophageal adenocarcinoma: an FNCLCC and FFCD multicenter phase III trial. J Clin Oncol 2011;29:1715-1721.

11 Al-Batran SE, Hofheinz RD, Pauligk C, et al: Histopathological regression after neoadjuvant docetaxel, oxaliplatin, fluorouracil, and leucovorin versus epirubicin, cisplatin, and fluorouracil or capecitabine in patients with resectable gastric or gastro-oesophageal junction adenocarcinoma (FLOT4-AIO): results from the phase 2 part of a multicentre, open-label, randomised phase 2/3 trial. Lancet Oncol 2016;17:1697-1708.

12 Hulscher JBF, van Sandick JW, de Boer AGEM, et al: Extended transthoracic resection compared with limited transhiatal resection for adenocarcinoma of the esophagus. N Engl J Med 2002;347:1662-1669.
Boshier PR, Anderson O, Hanna GB: Transthoracic versus transhiatal esophagectomy for the treatment of esophagogastric cancer: a meta-analysis. Ann Surg 2011;254:894-906.

14 Lewis I: The surgical treatment of carcinoma of the oesophagus; with special reference to a new operation for growths of the middle third. Br J Surg 1946;34:18-31.

15 Allum WH, Bonavina L, Cassivi SD, et al: Surgical treatments for esophageal cancers. Ann N Y Acad Sci 2014;1325:242-268

16 Blencowe NS, Strong S, McNair AG, et al: Reporting of short-term clinical outcomes after esophagectomy: a systematic review. Ann Surg 2012;255:658-666.

17 Luketich JD, Pennathur A, Awais O, et al: Outcomes after minimally invasive esophagectomy: review of over 1,000 patients. Ann Surg 2012;256:95-103.

18 Messager M, Warlaumont M, Renaud F, et al: Recent improvements in the management of esophageal anastomotic leak after surgery for cancer. EJSO 2017;43: 258-269.

19 Sisic L, Blank S, Nienhüser H, et al: The postoperative part of perioperative chemotherapy fails to provide a survival benefit in completely resected esophagogastric adenocarcinoma. Surg Oncol 2017;DOI: 10.1016/j.suronc.2017.06.001.

20 Denewer A, Fathi A, Setit A, et al: Totally endoscopic (thoracoscopic and laparoscopic) radical esophagectomy with gastric tube reconstruction through a small neck incision: an early experience with thirty Egyptian patients. Surgical Science;2014:5, article ID 46130.

21 Zhang R, Liu S, Sun H, et al: The application of singlelumen endotracheal tube anaesthesia with artificial pneumothorax in thoracolaparoscopic oesophagectomy. Interact Cardiovasc Thorac Surg 2014;19:308310.

22 Wolfer RS, Krasna MJ, Hasnain JU, McLaughlin JS: Hemodynamic effects of carbon dioxide insufflation during thoracoscopy. Ann Thorac Surg 1994;58:404408.

23 Perkins GD, Park D, Alderson D, et al: The Beta Ago nist Lung Injury TrIal (BALTI) - prevention trial protocol. Trials 2011;12:79:1-6.

24 Baudouin SV: Lung injury after thoracotomy. Br J Anaesth 2003;91:132-142.

25 Mao QX, Guo W, Huang B-Q, Yna H: Impact of artificial capnothorax on coagulation in patients during video-assisted thoracoscopic esophagectomy for squamous cell carcinoma. Surg Endosc 2016;30:2766-2772.

26 Shen $\mathrm{Y}$, Zhong M, Wu W, et al: The impact of tidal volume on pulmonary complications following minimally invasive esophagectomy: a randomized and controlled study. J Thorac Cardiovasc Surg 2013;146: 1267-1274.
27 Cuschieri A, Shimi S, Banting S: Endoscopic oesophagectomy through a right thoracoscopic approach. J R Coll Surg Edinb 1992;37:7-11.

28 Depaula AL, Hashiba K, Ferreira EA, et al: Laparoscopic transhiatal esophagectomy with esophagogastroplasty. Surg Laparosc Endosc Percut Tech 1995;5:1-5.

29 Cuschieri A: Thoracoscopic subtotal oesophagectomy. Endosc Surg Allied Technol 1994;2:21-25.

30 Palanivelu C, Prakash A, Rangaswamy S, et al: Minimally invasive esophagectomy: thoracoscopic mobilization of the esophagus and mediastinal lymphadenectomy in prone position. An experience of 130 patients. J Am Coll Surg 2006;203:7-16.

31 Luketich JD, Alvelo-Rivera M, Buenaventura PO, et al: Minimally invasive esophagectomy: outcomes in 222 patients. Ann Surg 2003;238:486-494.

32 Jafari MD, Halabi WJ, Smith BR, et al: A decade analysis of trends and outcomes of partial versus total esophagectomy in the United States. Ann Surg 2013:258: 450-458.

33 Luketich JD, Pennathur A, Franchetti Y, et al: Minimally invasive esophagectomy: results of a prospective phase II multicenter trial-the eastern cooperative oncology group (E2202) study. Ann Surg 2015;261:702707.

34 Biere SS, van Berge Henegouwen MI, Maas KW, et al: Minimally invasive versus open oesophagectomy for patients with oesophageal cancer: a multicentre, openlabel, randomised controlled trial. Lancet 2012;379: 1887-1892.

35 Verhage RJJ, Hazenbroek EJ, Boone J, van Hillegersberg R: Minimally invasive surgery compared to open procedures in esophagectomy for cancer: a systematic review of the literature. Minerva Chirurgica 2009;64: 135-146.

36 Li J, Shen X, Tan L, et al: Is minimally invasive esophagectomy beneficial to elderly patients with esophageal cancer? Surg Endosc 2015;29:925-930.

37 Straatman J, van der Wielen N, Cuesta MA, et al: Minimally invasive versus open esophageal resection: threeyear follow-up of the previously reported randomized controlled trial: the TIME trial. Ann Surg 2017;266: 232-236.

38 Messager M, Pasquer A, Duhamel A, et al; FREGAT working group: Laparoscopic gastric mobilization reduces postoperative mortality after esophageal cancer surgery: a French nationwide study. Ann Surg 2015; 262:817-822.

39 Mariette C, Markar S, Dabakuyo-Yonli TS, et al: MIRO trial: 3-year outcomes favor laparocopic surgery for oesophageal Cancer. ESMO 2017 Press Release. 
40 Takeuchi H, Miyata H, Ozawa S, et al: Comparison of short-term outcomes between open and minimally invasive esophagectomy for esophageal cancer using a nationwide database in Japan. Ann Surg Oncol 2017; 24:1821-1827.

41 Weksler B, Sullivan JL: Survival after esophagectomy: a propensity-matched study of different surgical approaches. Ann Thorac Surg 2017;104:1138-1146.

42 Gutschow CA, Schmidt H, Moons J, et al: Defining benchmarks for transthoracic esophagectomy - a riskadjusted multicenter analysis of 770 total minimally invasive esophagectomies; ESA 2017 abstract, $w w w$. esa2017.ro/download-files/ulLKfXe.pdf.

43 Glatz T, Marjanovic G, Kulemann B, et al: Hybrid minimally invasive esophagectomy vs. open esophagectomy: a matched case analysis in 120 patients. Langenbeck Arch Surg 2017;402:323-331.

44 Bjelovic M, Babic T, Spica B, et al: Could hybrid minimally invasive esophagectomy improve the treatment results of esophageal cancer? EJSO 2016;42:1196-1201.

45 Palazzo F, Rosato EL, Chaudhary A, et al: Minimally invasive esophagectomy provides significant survival advantage compared with open or hybrid esophagectomy for patients with cancers of the esophagus and gastroesophageal junction. J Am Coll Surg 2015:220: 672-679.

46 Bonavina L, Scolari F, Aiolfi A, et al: Early outcome of thoracoscopic and hybrid esophagectomy: propensitymatched comparative analysis. Surgery 2016;159: 1073-1081.

47 Broeders IA, Ruurda JP: Robotics in laparoscopic surgery: current status and future perspectives. Scand J Gastroenterol 2002;236(suppl):76-80.
Horgan S, Berger RA, Elli EF, Espat NJ: Robotic-assisted minimally invasive transhiatal esophagectomy. Am Surg 2003;69:624-626.

49 Kernstine KH, DeArmond DT, Karimi M, et al: The robotic, 2-stage, 3-field esophagolymphadenectomy. J Thorac Cardiovasc Surg 2004;127:1847-1849.

50 Van Hillegersberg R, Boone J, Draaisma WA, et al: First experience with robot-assisted thoracoscopic esophagolymphadenectomy for esophageal cancer. Surg Endosc 2006;20:1435-1439.

51 Ruurda JP, van der Sluis PC, van der Horst S, van Hillegersberg R: Robot-assisted minimally invasive esophagectomy for esophageal cancer: a systematic review. J Surg Oncol 2015;112:257-265

52 Cerfolio RJ, Wei B, Hawn MT, Minnich DJ: Robotic esophagectomy for cancer: early results and lessons learned. Semin Thoracic Surg 2016;28:160-169.

53 Abbott A, Shirdhar J, Hoffe S, et al: Robotic assisted Ivor Lewis esophagectomy in the elderly patient. J Gastrointest Oncol 2015;6:31-38.

54 Salem AI, Thau MR, Strom TJ, et al: Effect of body mass index on operative outcome after robotic-assisted Ivor-Lewis esophagectomy: retrospective analysis of 129 cases at a single high-volume tertiary care center. Dis Esophagus 2017;30:1-7.

55 Van der Sluis PC, Ruurda JP, Verhage RJJ, et al: Oncologic long-term results of robot-assisted minimally invasive thoraco-laparoscopic esophagectomy with twofield lymphadenectomy for esophageal cancer. Ann Surg Oncol 2015;22:S1350-1356.
6 Egberts J-H, Stein H, Anselmann H, et al: Fully robotic da Vinci Ivor-Lewis esophagectomy in four-arm technique-problems and solutions. Dis Esophagus 2017;30: 1-9.

7 Park S, Hwang Y, Lee HJ, et al: Comparison of robotassisted esophagectomy and thoracoscopic esophagectomy in esophageal squamous cell carcinoma. J Thoracic Dis 2016;8:2853-2861.

58 Kim DJ, Park SY, Lee S, et al: Feasibility of a robotassisted thoracoscopic lymphadenectomy along the recurrent laryngeal nerves in radical esophagectomy for esophageal squamous carcinoma. Surg Endosc 2014;28:1866-1873.

59 Park SY, Kim DJ, Do YW, et al: The oncologic outcome of esophageal squamous cell carcinoma patients after robot-assisted thoracoscopic esophagectomy with total mediastinal lymphadenectomy. Ann Thorac Surg 2017;103:1151-1157.

60 Hodari A, Park KU, Lace B, et al: Robot-assisted minimally invasive Ivor Lewis esophagectomy with realtime perfusion assessment. Ann Thorac Surg 2015;100: 947-953.

61 Mori K, Yoshimura S, Yamagata Y, et al: Preclinical study of transcervical upper mediastinal dissection for esophageal malignancy by robot-assisted surgery. Int J Med Robotics Comput Assist Surg 2017;13

62 Mori K, Yamagata Y, Aikou S, et al: Short-term outcomes of robotic radical esophagectomy for esophageal cancer by a nontransthoracic approach compared with conventional transthoracic surgery. Dis Esophagus 2016;29:429-434. 